\title{
Capital Cultural y Educación superior; una ecuación imperfecta ${ }^{1}$
}

\author{
Sonia Brito Rodríguez*
}

\begin{abstract}
Resumen
Este documento procura analizar la temática de capital cultural y dar cuenta de cómo ayuda a comprender las dinámicas de inserción y formación que tienen los estudiantes que ingresan a la educación superior, más aún cuando son la primera generación en ingresar a este nivel de educación. Interesa preguntarse sobre el potencial predictivo de permanencia académica del capital cultural.

Para abordar el concepto de Capital Cultural, es pertinente recoger los planteamientos que hace Bourdieu, a partir de algunos de sus estudios académicos que vinculan el capital cultural y el desempeño de los estudiantes en la educación elemental y posteriormente, con las posibilidades de obtener resultados positivos a nivel de educación superior. Estas investigaciones al momento de tratar de explicar las diferencias en el rendimiento académico de los estudiantes, evidencian que existen ciertas características que identifican y explican, en parte importante, el déficit de ciertas competencias académicas, las que inciden en sus capacidades de ingreso y permanencia en el sistema de educación superior.
\end{abstract}

Palabras clave: Capital cultural- educación superior- rendimiento académico

\section{Cultural capital and higher education; an imperfect equation}

\begin{abstract}
This paper is intended to examine the issue of cultural capital and how it helps to understand the dynamics of integration and training in students entering higher education, especially when they are the first generation to enter this level of education. It is worth wondering about the predictive potential of cultural capital in respect to academic permanence.

In order to address the concept of cultural capital, it is appropriate to adopt Bourdieu's approaches and some of his academic studies linking cultural capital and student achievement in elementary education and later, the potential for positive outcomes in higher education. In trying to explain differences in academic performance of students, these researches show there are certain characteristics that identify and largely explain the deficit of some academic skills that affect their ability to enter and remain in the higher education system.
\end{abstract}

Keywords: Cultural capital - higher education - academic performance

I Este trabajo forma parte de la tesis doctoral DIVERSIDAD CULTURAL, INCLUSIÓN E INTERCULTURALIDAD ENEDUCACIÓN SUPERIOR. Un estudio de los discursos y propuestas formativas en instituciones Universitarias no selectivas acreditadas e inclusivas de la Región Metropolitana.

* Candidata a Doctora en Ciencias de la Educación, mención educación intercultural, de la Universidad de Santiago de Chile. sbritor@ucsh.cl 


\section{La Herencia del Capital Cultural}

La familia se constituye en el agente socializador más relevante para los niños y niñas, siendo la responsable de la transmisión de la cultura primaria, que consiste en inculcar las normas, valores, pautas de comportamiento que les permitirán compartir formas de vida, y negociar lenguajes en la sociedad.

Las características asociadas a la procedencia familiar, tales como raza, etnia, religión, nivel socioeconómico, formas de hablar, se incorporan en el transcurso de la vida, y su influencia permanecerá e incidirá en las posibilidades de acceso u obstáculos en circuitos presentes y futuros de poder. Existen otros significantes tales como el entorno social, y la escuela, quienes aportarán en reforzar una posición social, así estos ambientes generan circuitos culturales acotados y apropiados de acuerdo a si son los herederos o no lo son.

Por tanto el éxito y la posición son heredadas, vinculándose a la asignación desigual de capital cultural. Pierre Bourdieu plantea que es un "instrumento privilegiado de la sociedad burguesa que confiere a los privilegiados el privilegio supremo de no aparecer como privilegiados", mientras que "logra tanto más fácilmente convencer a los desheredados de que deben su destino escolar y social a su falta de dones o de méritos cuanto más la desposesión absoluta excluya en materia de conciencia de desposesión" (Bourdieu y Passeron, I996:269).

En el mismo sentido, la educación no reduce las desigualdades educativas, sociales; esto produciría una perpetuación de clase social y su estratificación laboral. Atria plantea que "la literatura indica que la expansión del sistema educacional en sí misma no necesariamente reduce las desigualdades de clase en cuanto a las oportunidades de logro educativo" (Atria, 2004:39). Estas diferencias son las que indaga el concepto de capital cultural.

El Capital Cultural es un concepto instaurado y desarrollado por el sociólogo francés Pierre Bourdieu (1979); lo entiende como un instrumento de poder al nivel del individuo bajo la forma de un conjunto de cualificaciones intelectuales producidas por el medio 
familiar y el sistema escolar. Es un capital porque se puede acumular a lo largo del tiempo y también, en cierta medida, la transmisión a sus hijos, la asimilación de este capital en cada generación es una condición de la reproducción social. En tanto capital, concede poder a quien lo sustenta. Bourdieu señala que "la sociedad humana se asemeja a una competencia feroz cuyo premio es la posición social; la que se obtiene a través de acciones específicas como poseer capital económico, capital social y capital cultural. (Bourdieu, 2000).

Representa las formas de hablar, de actuar, los modos de vida, los movimientos, las socializaciones, las formas de conocer, las prácticas de lenguaje, los conocimientos, valores, principios, ideologías u otros que poseen un individuo o un grupo. Es distintivo y es un reflejo de la posición social, por tanto tiene directa relación con el capital económico, social y humano; pero que no es una condición determinante y definitiva, porque puede ser adquirida a través de la educación. Por lo tanto, capital cultural no es estático sino que varía, es dinámico en el tiempo, se acumula en la persona que lo porta durante toda su vida. Para Bourdieu y Passeron, "particularmente la enseñanza de la cultura (incluso científica), presupone implícitamente un cuerpo de saberes, de saber-hacer y sobre todo de saber-decir que constituye el patrimonio de las clases cultivadas" (Bourdieu, P. y Passeron, J.C., I964).

Desde la perspectiva de Bourdieu, la más oculta y determinada de las inversiones educativas, es la que radica en la transmisión o transferencia del capital cultural, que junto al capital económico, es el origen irrefutable del poder político y de la hegemonía social, que se reproduce hereditariamente materializando el poder y los privilegios mantenidos por las oligarquías de la clase dominante.

Para Bourdieu (1979), el capital cultural puede existir bajo tres formas:

El capital cultural es un tener transformador en ser, una propiedad hecha cuerpo que se convierte en una parte integrante de la "persona”, un hábito. Por ello goza de algunas características particulares, como por ejemplo que no puede ser transmitido instantáneamente, puede adquirirse de manera 
totalmente encubierta e inconsciente y queda marcado por sus condiciones primitivas de adquisición. No puede acumularse más allá de las capacidades de apropiación de un agente en particular; se debilita y muere con las capacidades biológicas de su portador; y quizá su característica más importante es que por su naturaleza, el capital cultural se transforma en capital simbólico, capital denegado o más bien desconocido.

Sin embargo, no hay que olvidar que este capital cultural solamente subsiste como capital material y simbólicamente activo, en la medida en que es apropiado por agentes y es comprometido, como arma y como apuesta que se arriesga en las luchas cuyos campos de producción cultural (artístico, científico, etc.) o más allá en el campo de las clases sociales, sean el lugar en donde los agentes obtengan los beneficios ganados por el dominio sobre este capital objetivado, y por lo tanto, en la medida en que su capital es incorporado.

En estado institucionalizado, el capital cultural se objetiva bajo la forma de títulos y se constituye en una de las maneras de neutralizar algunas de las propiedades que, por incorporado, tiene los mismos límites biológicos que su contenedor. Así por ejemplo es lo que ocurre con la graduación escolar y el posterior título profesional universitario o técnico. Es una especie de patente de competencia cultural que confiere a su portador un valor convencional, constante y jurídicamente garantizado desde el punto de vista de la cultura; la alquimia social produce una forma de capital cultural que tiene una autonomía relativa respecto de su portador y del capital cultural que él posee efectivamente en un momento dado.

Ciertamente, tal como sucede con muchas otras posesiones, el capital económico, social y cultural, no son poseídos por todas las personas en la misma medida, en este caso, entre otros aspectos, se confirma por las limitaciones en el acceso al consumo de bienes de capital cultural.

Se requiere de un cierto tiempo para la adquisición del capital cultural y luego para establecer el vínculo con el capital económico. Las diferencias entre el capital cultural de una familia, implican en primer término diferencias en la precocidad del inicio de su transmisión y acumulación. En segundo término, implica diferencias 
en la capacidad de satisfacer las exigencias propiamente culturales de una adquisición prolongada.

Y es en la lógica de la transmisión del capital cultural objetivado donde reside el principio más poderoso de la eficacia ideológica de este tipo de capital. Se sabe que la apropiación del capital cultural objetivado, por una parte, depende principalmente del capital cultural incorporado a la familia en su conjunto. Y por otra parte, que la acumulación inicial de capital cultural, comienza muy temprano, desde su origen, sin retraso ni pérdida de tiempo, solamente para aquellas familias dotadas con un fuerte capital cultural, lo cual les permite una acumulación más fácil y expedita de cualquier tipo de capital cultural útil. En cualquier caso, el tiempo de acumulación comprende la totalidad del tiempo de socialización; esto explica por qué la transmisión del capital cultural es la forma más disimulada de transmisión hereditaria de capital y, por lo mismo, su importancia relativa en el sistema de las estrategias de la reproducción es mayor en la medida en que las formas directas y posibles de transmisión de dicho capital, tienden a ser más fuertemente censuradas y controladas.

Lo más probable es que todas las culturas cuenten con sus propias arbitrariedades culturales. En consecuencia, con el proceso de socialización también se adquieren y se propagan tales arbitrariedades culturales. Realmente, en una sociedad segmentada o dividida en clases coexisten distintas culturas o subculturas. En lo que corresponde al sistema educativo, también contiene sus propias arbitrariedades culturales, que obviamente son las arbitrariedades impuestas por las clases dominantes.

Por tanto, los grupos más favorecidos por esta acumulación de ventajas -o sea, los herederos- deben a su origen no sólo "hábitos, entrenamiento y actitudes que les sirven directamente en sus tareas académicas", sino que "heredan también saberes y un saber-hacer, gustos y un 'buen gusto' cuya rentabilidad académica, aun siendo indirecta, no por eso resulta menos evidente" (Bourdieu y Passeron, 2003).

Los agentes sociales, a lo largo de su proceso histórico, sustentan un capital específico que ha acumulado, que es lo que cada individuo 
posee y ha interiorizado: capital social, capital económico y capital cultural. Además, al otorgarle un reconocimiento institucional al capital cultural poseído por un determinado agente, en forma de un título escolar o de una educación superior (que corresponde al capital cultural institucionalizado), les permite a sus titulares compararse, intercambiarse y establecer tasas de convertibilidad entre capital cultural y capital económico, garantizando así el valor monetario de un determinado capital escolar o de una educación superior.

El producto de la conversión del capital económico en capital cultural es el título, pues establece el valor relativo del capital cultural del portador de determinado título, en relación a los otros poseedores de títulos y también, de modo inherente, establece su valor de mercado, es decir, el valor en dinero por el cual puede ser cambiado en el mercado de trabajo. Y además de cierta forma define el tiempo en que un individuo prolongará su esfuerzo de adquisición, el que dependerá del tiempo libre que su familia de origen inicialmente le proveyera o que él mismo posteriormente puede asegurarse: como condición de la acumulación inicial, liberarse lo antes posible de la necesidad económica o bien, aumentar más aún el capital para después traspasarlo o heredarlo a su descendencia.

\section{Instalación del Capital Cultural en el Sistema Educativo}

La sociedad segrega, esto se internaliza al punto de establecer la tradición de que quien tenga más capital cultural (visto desde una hegemonía), estará en condiciones de superarse y acceder a mejor educación. Además, cómo éste, entre otros factores, influye en el acceso y éxito académico. Es así que las políticas que intentan corregir desigualdades operan desde lo compensatorio.

El potencial explicativo del concepto de capital cultural es tan amplio, que no sólo en el sentido anteriormente señalado se relaciona con la educación superior. En recientes investigaciones y artículos (Noble y Davies, 2009; Williams y Filippakou, 2009; Longden, 2004) ha sido utilizado dicho concepto como marco de referencia para analizar diversos temas, como por ejemplo, la variación de la 
participación en la educación superior, la formación de élites en la educación superior, y la deserción de estudiantes de las instituciones de educación superior.

En Longden (2004) se encuentra otro aporte importante que da cuenta de la relación entre el capital cultural y la educación superior. Este autor sugiere un modo de visualización de los datos de retención estudiantil mediante un marco complementario alternativo, basado en el concepto de capital cultural. El uso de este marco proporciona una forma útil de ver e interpretar los datos, donde parece ser importante la influencia de factores socioeconómicos. Además, proporciona un modo distinto de considerar la importancia de la experiencia educativa, la preparación a una nueva actividad o esfera social a través de una adaptación previa y realizar una inducción a los estudiantes, es decir, la consideración de su "habitus". La premisa que se utiliza se basa en la opinión de que todas las organizaciones tienen capital cultural que, en el caso de la educación superior, puede basarse en la creencia de los estudiantes sobre el estado del grado otorgado a ellos, el grado de dificultad para la admisión y la percepción más amplia de la situación de la institución. El "habitus" del estudiante establece el nivel de capital cultural disponible a través de las experiencias familiares y de la reproducción social.

El concepto de capital cultural debería ocupar un lugar central en la investigación educativa, puesto que explica las trayectorias académicas de los estudiantes de educación superior. Sin embargo no siempre es así, ya que raramente se le menciona en las políticas educativas, tales como: política de equidad, política de género, política de acceso, entre otros, donde se le utiliza como oposición al desmesurado interés por un concepto de capital humano de amplia utilización en los documentos de dicha política educativa.

A diferencia del capital humano ${ }^{2}$, el concepto de capital cultural se relaciona tanto a la educación desde el exterior como con los

2 En este enfoque se establece la productividad de la educación a través de la rentabilidad de invertir en formación. "El método más utilizado para demostrarla es el cálculo de los rendimientos privados o directos de las inversiones en educación, mediante la determinación de las diferencias de ingresos en el conjunto de la vida activa de individuos con distintos niveles de educación" (Feito, 200I). 
procesos que definen lo que serán los resultados educativos. La labor universitaria entonces es entrar en este proceso y actuar sobre el proceso mismo mediante el aporte cultural. Sin duda que el capital cultural ocupa un lugar muy importante debido a las variadas relaciones existentes y sus múltiples repercusiones en la educación, en las mayores habilidades que un estudiante pueda desarrollar y en la máxima potenciación de su desempeño.

La noción de capital cultural en educación superior presenta su mayor poder explicativo cuando se asocia a las nociones de habitus, estructuras y prácticas. Conceptos que son parte integrante del cuerpo teórico del que se desprende la primera noción. Como marco de referencia investigativo, el uso del concepto de capital cultural no puede desprenderse de su adecuada operatividad, de la búsqueda de las dimensiones, parámetros, variables, categorías e indicadores que mejor describan o representen sus reales manifestaciones.

Es posible señalar que los estudiantes más favorecidos aportan hábitos, modos de comportamiento y actitudes de su medio social de origen que facilitan su trayectoria académica, en cambio los que poseen un capital cultural distinto al dominante tendrían menos posibilidades de éxito en este tránsito. (Ezcurra, 2007; Tinto, V., 2005).

\section{Capital Cultural y Realidad Educativa como Distribuidor Segregador de Capital Cultural}

El concepto de capital cultural aparece asociado frecuentemente al desarrollo académico de los estudiantes en el sistema educativo; se habla de más o menos capital cultural para caracterizar a los estudiantes o se vincula como un factor determinante en la permanencia de éstos en el sistema. En este documento se realizará una revisión del concepto de capital cultural y del uso que de este término se observa en investigaciones en educación superior, intentando responder a la pregunta sobre su incidencia en el acceso y permanencia de los estudiantes en el sistema.

En cada una de las investigaciones, a través del concepto de capital cultural se han identificado distintas categorías de análisis. Entre ellas se pueden destacar las utilizadas por Noble y Davies (2009) quienes 
citando a Lamont y Lareau (I988) identifican cuatro modos por medio de los cuales el capital cultural puede operar para excluir a individuos de determinadas actividades y esferas sociales, tales como la educación superior. Los autores sostienen que en primer lugar, puede haber "self-elimination" ("autoeliminación"), por la cual los estudiantes reconocen anticipadamente que no coincidirán con la cultura de las instituciones de educación superior y por lo tanto es un lugar al que no pertenecen realmente o interpretan que los problemas que deberán superar, debido a su bajo capital cultural, hacen inútil o demasiado incierto participar en este ámbito.

Sin embargo, puede causar, producto de lo anterior, una "overselection ("sobreselección"), que indica que los estudiantes intentan superar sus desventajas culturales, pero no lo logran, ya sea porque no pueden completar una prueba o una aplicación, fallan en una prueba o en su aplicación o abandonan prematuramente al poco tiempo después de haberse matriculado. En tercer lugar, puede haber "relegation" ("relegación"), por la cual algunos estudiantes toman decisiones mal informadas, malinterpretadas, o en su defecto por tener escaso acceso a información pertinente. En este caso, el bajo capital cultural reduce la probabilidad de ingreso y permanencia en la educación superior porque los estudiantes infravaloran o minimizan los beneficios netos que recibirían con la misma. Finalmente, puede haber "direct selection" ("selección directa"), en la que existe arbitrariedad o parcialidad manifiesta por parte de aquellos académicos encargados de examinar las aplicaciones, y deciden quiénes ingresan o no a la universidad.

Chile ha experimentado en las últimas 3 décadas una vertiginosa expansión de la cobertura de la educación terciaria, "mientras en el año I983 había I75.250 jóvenes en la educación superior, el año 2012 esta cifra llegó a I.I27.I8I estudiantes” (SIES, 2012).

Este acelerado crecimiento de la matrícula generó un aumento de la cobertura bruta, la que, de acuerdo a la encuesta CASEN, pasó de I5,6\% en I990 a 45,8\% el 20II. (CASEN, 20I I).

Las trasformaciones de la educación superior más comúnmente reconocidas, se refieren principalmente a su masificación y diversidad, 
lo que ha implicado la llegada de un importante número de estudiantes que son la primera generación con estudios universitarios en su familia; "... de una relación estrecha con los sectores altos en los años ochenta e inicios de los noventa, se ha pasado paulatinamente a una vinculación con sectores socioeconómicos más vulnerables, de menores ingresos incluso que los tradicionalmente integrados en el sistema tradicional de universidades”. (Calderón, V., 20II).

Según la OECD (20II), el crecimiento del sistema ya no está en el centro de la discusión pública. En su lugar han surgido con fuerza temas como la equidad en el acceso, la calidad de las instituciones de educación superior y el papel que deben jugar en la sociedad.

Dicha masificación supuso cambios en la composición del alumnado, con la entrada de clases sociales desfavorecidas -además de otros sectores, como indígenas y personas del medio rural, entre otros(Rama, C., 2006b). Lo anterior ha puesto de relieve el concepto de capital cultural en tanto su posesión o ausencia incidiría en el logro educativo en los distintos niveles de enseñanza.

La educación es la herramienta privilegiada de reproducción social, es decir, del mantenimiento del orden social según la más antigua tradición cultural. Bajo esta perspectiva vale considerar algunas reflexiones donde Bourdieu, P. (2003) puede aportarnos algunas luces para interpretar esta realidad.

Esta arbitrariedad cultural se transmite a la educación, entendiendo que son las clases dominantes quienes deciden las propuestas educativas; los alcances de los contenidos, y las metodologías. El qué y cómo enseñar. Desde los estudios de Bourdieu, el resultado de esto, los estudiantes pertenecientes a las clases dominantes, a diferencia de niños y jóvenes de las clases dominadas, encuentran inteligible y asequible la educación que se les inculca, al estar más cercana a los códigos y modos practicados por las familias de las clases dominantes.

En primer lugar existe una representación dominante en el discurso universitario, donde los profesores suponen que los alumnos son como ellos fueron y confunden frecuentemente "sus tiempos" con 
los de la actualidad; hay en este sentido una confusión generacional por incomprensión del presente. En segundo lugar, hay un conjunto de iniciativas, políticas e instrumentos de evaluación, proyectos pedagógicos y diseños curriculares que se establecen bajo la consideración de un alumno ideal, al que deben ajustarse los alumnos reales; se transmite una visión del alumno exitoso que responde a un perfil determinado, lo que corresponde propiamente a un acto de violencia simbólica. (Casillas, M.; Chain, R.; Nancy Jácome, N., (2007).

Desde inicios de los '90, diversas investigaciones mostraron que los profesores secundarios en el aula constituyen la variable institucional más relevante en el desempeño estudiantil, tan decisiva que su incidencia es mayor que cualquier otro factor del establecimiento (Astin, A., I993; Evenbeck, S. y Jackson, B., 2005). El docente es un actor relevante en el proceso de transmisión y/o distribuidor de capital cultural, puesto que se constituye en un nexo entre los aprendizajes que se ponen en práctica y llegan al aula y sus mediaciones axiológicas, ideológicas, entre otras.

Es en este sentido que la acción o actividad de un docente es relevante, puesto que va más allá de ser un mero "transmisor de contenidos por hora"; es la apuesta por la identidad lo que permite denominarlo un académico: la construcción del intelectual cuyo punto en común es su pertenencia a las instituciones educativas y su participación en las funciones de producción y transmisión del conocimiento (Preciado, F., 2006).

El valor del académico se basa en su capacidad de distribuir capital cultural, a partir de sus tres estados: capital cultural en estado incorporado, capital cultural en estado objetivado y capital cultural en estado institucionalizado (Bourdieu, 1987). Cómo transfiere capital cultural el docente a los que se relacionan con él y que requieran adquirirlo. Este capital cultural es parte del habitus del docente universitario o de una institución de educación superior, un elemento necesario que ocupa un lugar determinado en un campo cultural y del saber, como es el campo educativo. Corresponde a esquemas mentales y prácticos incorporados, de visiones y divisiones sociales objetivas, 
que configuran principios de diferencia y pertenencia, que lo hacen ser reconocido como un profesor universitario o de una institución de educación superior. (Bourdieu y Waqcuant, 1995).

Es posible señalar que los grupos más favorecidos aportan hábitos, modos de comportamiento y actitudes de su medio social de origen que facilitan su trayectoria académica, en cambio los que poseen un capital cultural distinto al dominante tendrían menos posibilidades de éxito en este tránsito. (Ezcurra, 2007; Tinto, V., 2005).

Una de las manifestaciones del capital cultural es el capital social.

Es posible abordar las distintas formas del concepto de capital social desde dos dimensiones o ejes principales. La primera lo entiende como una capacidad específica de movilizar recursos por parte de un grupo, y la segunda se remite a la disponibilidad de redes de relaciones sociales (Atria, 2003). En otro sentido, Bourdieu, P. (I985) plantea que capital social "refiere al conjunto de recursos reales o potenciales a disposición de los integrantes de una red durable de relaciones más o menos institucionalizadas”.

Es frecuente que el concepto de capital social esté reducido a la consideración del nivel socioeconómico de los padres. Casillas, M.; Chain, R.; Nancy Jácome, N. (2007), plantean que” renunciamos de manera expresa a esta reducción analítica, para nosotros el acceso a los bienes culturales está mediado por los recursos que los agentes sociales tienen para apropiárselos, de ahí que el factor económico sea importante, pero no único, ni funciona en tanto capital económico exclusivamente, para favorecer dicha apropiación”. Los mismos autores señalan que "no se trata de negar la existencia de diferencias económicas entre los individuos y grupos sociales, sino de observar que éstas, en el terreno específico de la cultura y la educación se expresan a través de recursos y disposiciones culturales incorporadas en los individuos".

Según Sudarsky (200I), el capital social puede ser generado en diferentes instancias y su desarrollo depende de los valores, las costumbres, las creencias y la cultura de la sociedad. En consecuencia, la educación desempeña un papel muy importante, pues durante los 
diferentes niveles de formación de la persona, se pueden desarrollar algunas de las características que favorecen la creación de capital social. (En Díaz de Iparraguirre, 2008).

En nuestros días, el paso por la escuela (la permanencia, los promedios, los grados) es un indiscutible principio de diferenciación social. Las ofertas de trabajo se estructuran de acuerdo con determinados diplomas y certificados, las posiciones de mérito se asocian a la alta escolaridad y los mejores salarios los obtienen (normalmente) los más calificados. (Casillas; Chain; Jácome, 2007).

\section{Realidad Educativa y el Capital Otro}

Nuestra socialización ha estado vulnerada por prácticas autoritarias, en la familia, en la escuela, en el Estado, etc. Esto ha provocado que nuestra identidad tienda a ser homogeneizadora, discriminadora, excluyente, temerosa, invisibilizada.

Existe un deber ser aceptado, de patrones establecidos, que paraliza la curiosidad y limita la búsqueda. Se adiestra para repetir patrones probados. Paulo Freire hace alusión a la educación bancaria, cuando plantea que el estudiante es sólo depositario de conocimiento, nos conformamos con fórmulas, que aun cuando ineficientes, están probadas.

La Unesco plantea que aunque necesario, ya no es suficiente que los estudiantes desarrollen sus capacidades de naturaleza cognitiva y aprendan las destrezas básicas para acceder a un trabajo digno y gratificante. También es preciso que desplieguen habilidades sociales para convivir en un mundo plural, que logren un desarrollo afectivo equilibrado y una conciencia moral que les permita actuar con autonomía y responsabilidad. (UNESCO, 1996).

Para fortalecer este discurso se requiere generar mediaciones en el aprendizaje; esto necesariamente requiere cambiar el concepto de enseñanza-aprendizaje tradicional, e instalar los espacios socialesacadémicos en un ambiente para todos. Requiere un cambio de mirada, desde la política pública, que permita un viraje epistémico, de campos semánticos y relacionales; es decir, resituar y relevar otras inteligencias, tales como: inteligencias culturales, interpersonales, 
artísticas, sociales, entre otras. Ese salto cualitativo - de la instrucción a la formación- permitiría ir avanzando a una educación con sentido, en la formación de personas, estudiantes, ciudadanos.

El desafío entonces es el enriquecimiento de pensamiento, ampliar horizontes de la razón, lo dado y no dado, lo producido y lo potencial, lo que debe ser resignificado por el (los) sujeto(S), que participan en la educación.

El reconocer a los jóvenes desde su complejidad, multiplicidad, diversidad; intelecto y sentidos, pensamiento y sentimiento, que propicien la construcción de nuevos relatos, visión del mundo y del ser humano diferentes. Esta necesidad de construir requiere de ciertos soportes para ir avanzando hacia la comprensión, la amabilidad, la empatía, contrarrestando ciertas lógicas ciegas de discriminación, exclusión, pobreza.

El despliegue de etas destrezas y potencialidades cognitivas, sociales y valóricas que se aprecia en los jóvenes que ingresan a la educación universitaria son diversas. A menudo estas habilidades son subvaloradas, si no responden al patrón de la hegemonía, o estándar.

De Garay plantea que "Los jóvenes que logran ingresar a las instituciones de educación superior, como lo muestran distintos estudios, no son un grupo homogéneo. Provienen de diferentes estratos sociales, de diferentes ambientes culturales y familiares y tienen diferentes expectativas e imaginarios sobre la educación superior" (De Garay, 200I).

Es imprescindible desprenderse de los estereotipos culturales y sociales, que han colonizado y naturalizado nuestras consideraciones y acciones respecto de un deber ser relacional; debemos cambiar la mirada, pensar de "otra manera"; la Universidad abre ese camino, pues permite ir recorriendo otros senderos epistémicos, y semánticos.

La Universidad, por tanto, se constituye en ese puente ético, protagónico, en donde se manifieste con mayor naturalidad la reciprocidad, el reconocimiento, el respeto, puesto que es allí donde conviven el conocimiento, el pensamiento y la afectividad. 
Lo que realmente importa si se quiere formar ciudadanos éticos, comprometidos, integrales, es que podamos aprender a vivir juntos. Es indudable que habrá que repensar la noción de sujeto pedagógico. Pero depositar las esperanzas intactas, supone vislumbrar avances en la virtud de la cooperación en educación. Por ahí ha de emprenderse la reconversión del oficio de educar, pues es lo único que puede permitirnos reconstruir el mundo que compartimos con los demás.

\section{Discusiones}

La presión de la demanda de la educación superior contribuyó a la expansión y diversificación de la oferta y esto, a su vez, permitió el ingreso de nuevos contingentes sociales y culturales que fueron cambiando el perfil social del estudiantado. Hasta mediados del siglo pasado, las universidades de América eran instituciones "de elite” a las que asistían un grupo selecto de la sociedad que reunía el mayor capital cultural, económico y social (Rezaval, J., 2008). Hoy en día el alumnado de la educación superior se caracteriza por su heterogeneidad social y su diversidad etaria, existiendo diversos perfiles en el sistema (PNUD-Mideplan, 2006).

Tradicionalmente, aunque no exista información desagregada respecto del alumnado de escasos recursos en la Educación Superior, se ha pronosticado una alta deserción respecto de ellos por los siguientes motivos: la inferior calidad del medio estudiantil anterior; · la falta de red social. Generalmente se trata de la primera generación que ingresa a la Universidad y no encuentra a sus pares en educación en el entorno cercano; el ingreso familiar insuficiente, y en la mayoría de los casos, la falta de apoyo de la Universidad considerando la situación real. (Arrau, F., 2009).

Esta sería una de las razones por las que los estudiantes de sectores populares experimentan problemas académicos, siendo una de las consecuencias la deserción. En esta dirección, Tinto asienta que en EE.UU. para muchos estudiantes de bajos ingresos la universidad se ha convertido en una puerta giratoria: así como entran, salen. Es importante señalar que los estudios demuestran que los mecanismos de selección a la Universidad, en tanto filtros de ingreso, tenderían 
a reproducir la selección social, por tanto la incorporación selectiva de los estudiantes a la universidad se transforma en un problema de índole social. (Tinto, V., 2005).

La universidad debería ser más que un expositor de materias, información o conocimientos técnico-teóricos; más que un certificador académico o un entregador de títulos profesionales o un formador de profesionales. Debería ser un "alma mater”, una guía ético-moral, que con disciplina prepara personas íntegras, integrales e integradas. En este mismo sentido, se debe preocupar de inculcar el habitus $y$, a su vez, los estudiantes incorporarlo internamente, aprendiéndolo mediante el cuerpo, mediante un proceso de familiarización práctica, que no pasa por la conciencia (es un acto reflejo, involuntario e inconsciente), para luego expresarlo en forma positiva y sin prejuicios, en la misma educación superior y a lo largo de su propia vida ("bonus habitus"). Sin embargo, el habitus también puede ser usado como un elemento prejuicioso y discriminador: el habitus permite que personas de un determinado entorno social homogéneo tiendan a compartir estilos de vida similares.

El sistema educativo actual, incluyendo el de la educación superior universitaria, perpetúa las arbitrariedades culturales, sociales y económicas y así persistirán indefinidamente a no ser que algo cambie; porque muchas de las personas que estudiaron, no reciben proporcionalmente los beneficios de su inversión de tiempo, esfuerzo y dinero, puesto que aunque tengan un poco más de capital cultural y social, el cambio cualitativo en la posición inicial es muy leve y a veces hasta nulo, porque ese capital adquirido finalmente lo usufructúan los mismos de siempre, aquellos que ostentan o son en parte dueños del poder económico, político y social, y que no permiten que el Estado sea quien se haga cargo del financiamiento total de la educación pública universitaria. Eso por qué, porque ahora también son dueños de universidades privadas. El capital adquirido lo usufructúan las clases privilegiadas, a través de sus empresas, pues pueden conseguir trabajadores educados, capacitados y a un bajo costo.

El capital cultural entendido como visiones del mundo, refiere a la imagen global de los seres humanos y de las normas que deben 
guiar los comportamientos de las personas. Es el marco global de las creencias básicas de uno con respecto a las cosas, tiene que ver con el modo en que cada sociedad determina su cultura particular: creencias, valores, ideas y percepciones sobre el entorno, etc.

El capital cultural entendido como visiones del mundo, refiere a la imagen global de los seres humanos y de las normas que deben guiar los comportamientos de las personas. Es el marco global de las creencias básicas de uno con respecto a las cosas, tiene que ver con el modo en que cada sociedad determina su cultura particular: creencias, valores, ideas y percepciones sobre el entorno, etc.

Es a través de la mayor proporción del capital cultural que poseen las clases dominantes, lo que les permite imponerse sobre las clases dominadas, instrumentalizando el sistema de enseñanza y el proceso educativo, para transmitir las arbitrariedades culturales, que le son funcionales para mantener su hegemonía y perpetuar sus propios privilegios.

Se entiende que el escaso capital cultural que tienen algunos estudiantes que ingresan a la educación superior, por ser de una posición social baja, debe ser adquirido en este nivel de enseñanza superior, ya que cada nivel educativo va agregando capital cultural al estudiante y lo que no se ha adquirido en los niveles inferiores de enseñanza, debe de alguna forma compensarse en la educación superior. Entonces, las instituciones que imparten este tipo de educación deben ser capaces de disponer de espacios físicos e intelectuales apropiados para que ello ocurra. Debe encargarse también por nivelar otros ámbitos, en particular la incorporación de un repertorio lingüístico: con los códigos sociolingüísticos formales.

Al ingresar a la educación universitaria, el capital cultural es muy relevante porque facilita a los estudiantes los resultados positivos en el quehacer académico. Esta situación va disminuyendo a medida que se avanza y se van superando los niveles en la carrera y en cuanto al capital social es importante siempre, más aún cuando el estudiante egresa y sale al campo laboral. Puesto que nuestra sociedad es clasista y segregadora, discrimina por sector social, raza, aspecto físico, edad, etc. Exige, abierta o solapadamente, cierto arquetipo de ser humano 
(se aprecia claramente en la publicidad) y con ello se pretende la permanencia del statu quo de las clases privilegiadas.

Es urgente hacer un giro ético-político, que permita romper con el determinismo que considera que los sectores sociales de los primeros quintiles, deben recibir una educación de exigencia mínima por el hecho de que no poseen el patrimonio material suficiente. Se hace necesario fundar una institucionalidad que considere la atención temprana, en los años iniciales de la vida de una persona.

\section{Conclusiones}

Finalmente, dado que la posesión y adquisición de capital cultural no puede verse de manera estática, sino como un proceso dinámico y en permanente cambio y evolución, las recientes investigaciones arrojan diferentes resultados, a veces hasta contradictorios, que dan indicios acerca de la importancia pero no así de la determinación del capital cultural sobre el desarrollo de las personas y las sociedades.

El capital cultural ocupa un lugar central en el ámbito educativo debido a las múltiples relaciones existentes y repercusiones de aquél en la educación, en las habilidades que un estudiante pueda desarrollar y en la máxima potenciación de su desempeño. En este sentido, la adquisición de nuevos babitus, la consideración de factores como el tiempo disponible para la adquisición y acumulación de capital cultural, las características del sistema educativo y las posibilidades de acceso al mismo por parte de todos los miembros de una sociedad, entre otras, podrían considerarse como variables relevantes a la hora de vincular este concepto con fenómenos o hechos propios de la educación superior.

Si bien no se puede dejar de reconocer que el sistema de educación superior ha ampliado las oportunidades para que los egresados de enseñanza secundaria que provienen de los estratos más vulnerables de la población continúen estudios superiores, la sociedad chilena ha mostrado su fuerte desigualdad (Sapelli, 2009, 2009 ${ }^{a}$ ), principalmente expresada en la alta predictibilidad que muestran sus resultados en la transmisión intergeneracional, siendo el factor "cuna de origen del estudiante" determinante sobre los resultados 
en el ciclo escolar y con mayor fuerza en el ingreso y permanencia en la educación superior; pese a que se han incorporado un número importante de estudiantes que son primera generación familiar en educación superior, este factor se asocia fundamentalmente a la expansión de las vacantes (OCDE, 2009; Donoso y Cancino, 2007). Quienes poseen al menos uno de sus padres con educación superior y con recursos para compensar las demandas de aprendizaje que no pudieron alcanzar en el ciclo secundario, suelen tener una alta probabilidad de ingresar a las universidades más prestigiosas y permanecer estudiando (Donoso, S.; 2003; 2008; Donoso, S. 2010).

Antecedentes provistos por la OCDE (2009) sugieren que las tasas de éxito educativo varían según puntaje en las pruebas de admisión y tipo de establecimiento educativo, variables fuertemente asociadas al estrato o nivel socioeconómico de los alumnos. Tal como lo indica la OCDE (2009), los problemas de deserción pueden explicarse en parte por la inequidad del sistema secundario, que proporciona una formación deficiente a los estudiantes de menores ingresos. (Canales, A. y De los Ríos D., 2009).

Los miembros de la clase dominante, en cambio, tienen la ventaja añadida de haber sido educados en la cultura a partir de la cual se deriva buena parte de la educación formal; es decir, ya están equipados con lo que Bourdieu denomina «capital cultural», que pueden invertir para cosechar más tarde los beneficios en términos de un alto rendimiento educativo. Pero a los miembros de las clases subordinadas les falta ese capital. (Littlewood, 2005).

Los autores estudiados coinciden en que los factores personales y académicos, familiares, determinan si un estudiante es exitoso o no al final de su carrera profesional. Bajo esta perspectiva, Tinto postula que los estudiantes ingresan a la universidad con diversas habilidades y patrones de características personales, familiares y académicas, incluidas metas y predisposiciones iniciales para asistir a la universidad. (Tinto, V., I995). Estas últimas se modifican y reformulan continuamente a través de una serie de interacciones entre el individuo y las estructuras y miembros de los sistemas sociales y académicos de la institución. Las interacciones con los sistemas 
formales e informales sociales y académicos que resultan satisfactorias y recompensantes promueven la mayor integración a estos sistemas y, por lo tanto, la retención del estudiante. (Méndez, I y otros 20I0).

Ante ello, se reclama un giro. Un viraje de políticas. Así, habría que apuntar a otro objetivo: una mejor educación $-y$ ya no a amenguar u obturar la deserción-. O sea, la retención no debería ser un propósito, sino un resultado (Crissman, J. y M. Upcraft, 2005). En otros términos, una mejor educación daría la llave de la persistencia. Por eso, V. Tinto (2006b) arguye que los programas de retención exitosos se enfocan invariablemente, primero y antes que nada, en la educación de los alumnos, sobre todo durante el primer año. (En Ezcurra A.M., 2007).

\section{Referencias bibliográficas}

Atria, Raúl (2003). "Capital social: concepto, dimensiones y estrategias para su desarrollo” en Atria, R. et al. (compiladores). Capital social y reducción de la pobreza en América Latina y el Caribe: en busca de un paradigma. CEPAL y Universidad de Michigan, Santiago de Chile.

Atria, R. (2004). Estructura ocupacional, estructura social y clases sociales, CEPAL: Serie Políticas Sociales No 96, Santiago: I-5I p.

Astin, A. W. (1993). Assessment for Excellence. Phoenix, AZ: American Council on Education and Oryx Press.

Bernstein, B. (I99I). "La perspectiva Sociolingüística y la transmisión cultural" en. El debate social en torno a la educación. Enfoques predominantes. Antología. UNAM/ENEP. Acatlán, México. DF.

Bourdieu, P. y Passeron, J.C. (I964). Les héritiers. Les étudiants et la culture. Paris: de minuit. pág 36

Bourdieu, P. y Passeron, J.C. (1977). Reproduction in Education, Society and Culture (London and Beverly Hills, Sage Publications).

Bourdieu, P. (1979). Los tres estados del capital cultural. Sociologica, UAMAzcapotzalco, No 5, pp. II-I7. México.

Bourdieu, P. (1988). Cosas dichas. Ed. Gedisa S.A. Buenos Aires, Argentina.

Bourdieu, P. (I99I). El sentido práctico. Ed. Taurus, Madrid, España.

Bourdieu, P. (1988). La distinción. Criterio y bases sociales del gusto, Madrid, Taurus, p. I08. 
Bourdieu, P. (I99I). La distinción. Criterio y bases sociales del gusto, Madrid, Taurus, p. 104.

Bourdieu, P. y Passeron J.C. (I996): la reproducción. Elementos para una teoría del sistema de enseñanza, Fontamara, Barcelona: 269.

Bourdieu, Pierre y Passeron, Jean Claude (2003). "La elección de los herederos", en Los berederos. Los estudiantes y la cultura. Buenos Aires: Siglo XXI, pp. I I-45.

Bourdieu, Pierre (1987). "Los tres estados del capital cultural", en Sociológica, año 2, núm. 5, UAM- Azcapotzalco.

Bourdieu, Pierre (1985 [1975]) "El lenguaje autorizado: las condiciones sociales de la eficacia del discurso ritual”, en ¿Qué significa bablar? Economía de los intercambios lingüísticos, Akal Universitaria.

Bourdieu, P. (2000). Sobre el poder simbólico. En Intelectuales, política y poder. UBA/Eudeba. Buenos Aires.

Bourdieu, P. (2002). Estructuras, hábitus, prácticas. En Bourdieu, P. (I99I). El sentido práctico. Taurus Ediciones. Madrid.

Bourdieu, P. (2003). Los herederos. Los estudiantes y la cultura. Buenos Aires: Siglo XXI.

Bourdieu, P. y Loï J. D. Wacquant (1995). Respuestas. Por una antropología reflexiva. México: Grijalbo.

Calderón. V. (20II). Foro AEQUALIS de Educación Superior - Centro de Investigación en Estructura Social, Universidad de Chile. Caracterización social de los estudiantes de educación superior en Chile. Primer informe preliminar - Aspectos estructurales.

Canales, A. y De los Ríos D. (2007). Factores explicativos de la deserción universitaria. Revista Calidad en la Educación, N²6 Consejo Nacional de Educación, pp. I73-20I ISSN 0717-4004.

Casillas, M.; Chain, R.; Nancy Jácome, N. (2007). Origen social de los estudiantes y trayectorias estudiantiles en la universidad veracruzana. Revista de la Educación Superior, abril-junio, año/vol. XXXVI (2), número I42 Asociación Nacional de Universidades e Instituciones de Educación Superior, Distrito Federal, México.

Centro de Estudios del Ministerio de Educación (2012). Inclusión del ranking en el proceso de admisión 2013: ¿Favorece realmente la equidad? Serie Evidencias, Año I, No 7.

Crissman, Jennifer y M. Lee Upcraft (2005). "The keys to first-year student persistent”, en Upcraft M. Lee, John N. Gardner, Betsy O. Barefoot $\&$ Associates. 
De Garay Sánchez, Adrián (200I). Los actores desconocidos. Una aproximación al conocimiento de los estudiantes. Colección Biblioteca de la Educación Superior. México: ANUIES.

Díaz de Iparraguirre, A.M. (2008). La responsabilidad social de la universidad en la promoción del capital social para el desarrollo sustentable, $<=$ "”, i=”” style="box-sizing: border-box; ">Edición electrónica gratuita. Texto completo en www.eumed.net/libros/2008b/402/

Donoso, S. (2009). Economía Política del Financiamiento de los Estudios Universitarios en Chile (1980-2010): Debate de sus fundamentos. Una versión preliminar del trabajo se presentó en el Primer Congreso Chileno de Educación Superior, Santiago, octubre de 2009, organizado por Centro de Políticas Comparadas en Educación Superior (U. Diego Portales).

Donoso, S. y Cancino, V. (2007). Caracterización Socioeconómica de los Estudiantes de Educación Superior. Calidad en la Educación N ${ }^{\circ} 26$, julio, pp. $203-244$.

Donoso, S.; Schiefelbein, E. (2007). Análisis de Los Modelos Explicativos de retención de estudiantes en la Universidad: Una visión desde la desigualdad social. Universidad de Talca.

Evenbeck, S.E. \& Jackson, B. (2005). Faculty Development and the First Year. En M. L. Upcraft, J. N. Gardner \& B. O. Barefoot (Eds.), Challenging and Supporting the First- Year Student, San Francisco, CA, 257-274.

Ezcurra, A. M. (2005). "Diagnóstico preliminar de las dificultades de los alumnos de primer ingreso a la Educación Superior”, en Perfiles Educativos, Vol. XXVII, N I07.

Ezcurra, A.N, (2007). Los estudiantes de nuevo ingreso: democratización y responsabilidad de las instituciones universitarias. Universidad Nacional de General Sarmiento Argentina.

Goicovic, I. (2000). «Del control social a la política social. La conflictiva relación entre los jóvenes populares y el Estado en la historia de Chile». Última Década NI2. Viña del Mar: Ediciones cidpa. [Links ]

Lamont, M. and Lareau, A. (1988). Cultural Capital: Allusions, Gaps and Glissandos in Recent Theoretical Developments. Sociological Theory, 6(2): I53-I68.

Littlewood, P. (2005). Escolarización exclusiva. Paradigmas de gobernación y de la exclusión social en la educación: fundamentos para el análisis de la discriminación escolar contemporánea. En: Luengo, Julián (Comp.). Barcelona, Ediciones Pomares, pp. 62-64. 
Longden, B. (2006). An Institutional Response to Changing Student Expectations and their Impact on Retention Rates. Journal of Higher Education Policy \& Management, XXVIII (2),I73-I87.

Longden, B. (2004). Interpreting student early departure form higher education through the lens of cultural capital. Kluwer Academic Publisher. Netherlands.

Méndez, I. y otros (2010). Variables asociadas al éxito académico en estudiantes de la Licenciatura en Medicina de la UNAM.

Noble, J. y Davies, P. (2009). Cultural capital as an explanation of variation in participation in higher educación. British Journal of Sociology of Education, 30:5, 59I-605.

OCDE (2009). La Educación Superior en Chile. Revisión de Políticas Nacionales de Educación.

OECD (20I I). Education at a glance 20II.

PNUD-Mideplan (2006) Expansión de la educación superior en Chile: Hacia un nuevo enfoque de la equidad y calidad. Temas de Desarrollo Humano Sustentable No IO. Disponible en: http://www. revistadesarrollohumano.org/temas3I.asp\#I

Preciado, F. (2006). La cultura académica de los profesores colimenses universitarios: Una revisión a partir de la entrevista de historia oral. Estudios sobre las Culturas Contemporáneas, XII (23).

Rama, C. (2006b). Los nuevos estudiantes, UDUAL / Universidad de Campinas / ANDIFES / IESALC.

Rezaval, J. (2008). Tesis: Políticas de inclusión social social en la educación superior en Argentina, Chile y Perú. Flacso: Argentina.

Sapelli, C. (2009). Los retornos de la educación. Chile: estimaciones por Corte Transversal y por Cohortes. Instituto de Economía, Pontificia Universidad Católica de Chile, Doc. de Trabajo $N^{\circ} 349$, enero.

Sapelli, C. $\left(2009^{\mathrm{a}}\right)$. The evolution of the intergenerational mobility of education in Chile by cohorts; facts and possible causes. Instituto de Economía, Pontificia Universidad Católica de Chile, Doc. de Trabajo $\mathrm{N}^{\circ} 348$, enero.

SIES (2012). Evolución de la matrícula total por tipo de institución (I983 - 2011).

Tinto, V. (1987). El abandono de los estudios superiores: Una Nueva Perspectiva de las Causas del Abandono y su Tratamiento. México: Universidad Nacional Autónoma de México. 
Tinto, V. (2003). Promoting Student Retention Through Classroom Practice. Presented at Enhancing Student Retention: Using International Policy and Practice. An international conference sponsored by the European Access network and the Institute for Access Studies at Staffordshire University. Amsterdam.

Tinto,V. (2005). Research and practice of student retention: what next?, Pell Institute for the Study of Opportunity in Higher Education, Washington.

Tinto, V. (2006). Moving from theory to action: Building a Model of Institutional Action for Student Success. National Postsecondary Education Cooperative.

Tinto, V. (2006b). Educational communities and student success in the first year of university, Monash University, Australia.

Tinto, V.; Russo, P.; Kadel, S. (I994). Constructing educational communities: increasing retention in challenging circumstances. Community College Journal, LXIV (4), 26-29.

Tinto V. (1995). Transition from secondary school to university. Presentation prepared at the Monash University Conference; Monash University, Melbourne Australia: 62-4.

Williams, G. y Filippakou, O. (2009). Higher education and UK elite formation in the twentieth century. Springer Science Business Media. 